\title{
Gradhiva
}

\section{Gérard Toffin, La Fête-Spectacle. Théâtre et rite au}

Népal

Paris, Éditions de la Maison des sciences de l'homme, 2010

David N. Gellner

\section{(2) OpenEdition}

\section{Journals}

Édition électronique

URL : http://journals.openedition.org/gradhiva/2231

DOI : $10.4000 /$ gradhiva.2231

ISSN : 1760-849X

\section{Éditeur}

Musée du quai Branly Jacques Chirac

Édition imprimée

Date de publication : 30 novembre 2011

Pagination : 267-269

ISBN : 978-2-35744-046-3

ISSN : 0764-8928

\section{Référence électronique}

David N. Gellner, «Gérard Toffin, La Fête-Spectacle. Théâtre et rite au Népal », Gradhiva [En ligne], 14 |

2011, mis en ligne le 30 novembre 2011, consulté le 21 septembre 2020. URL : http://

journals.openedition.org/gradhiva/2231; DOI : https://doi.org/10.4000/gradhiva.2231

Ce document a été généré automatiquement le 21 septembre 2020.

(c) musée du quai Branly 


\section{Gérard Toffin, La Fête-Spectacle. Théâtre et rite au Népal}

Paris, Éditions de la Maison des sciences de l'homme, 2010

David N. Gellner

\section{RÉFÉRENCE}

Gérard Toffin, La Fête-Spectacle. Théâtre et rite au Népal, Paris, Éditions de la Maison des sciences de l'homme, 2010, $204 \mathrm{p}$. 
1 En août 2008, Pushpa Kamal Dahal, dit Prachanda («le Féroce», «le Redoutable»), leader incontesté des maoïstes au Népal, devenait le Premier ministre du pays. Le plus grand succès des rebelles fut justement considéré comme un triomphe. Douze ans auparavant, les grands partis politiques n'avaient pas pris au sérieux ce qui n'était encore qu'un groupuscule pratiquement inconnu. En 1996, commençaient dix années de guerre civile au terme desquelles les combattants maoïstes, armés à l'origine de couteaux et de quelques vieux fusils pris à la police, parvenaient à faire jeu égal avec l'Armée royale népalaise - même s'ils n'ont jamais été en mesure de s'emparer d'une ville plus longtemps qu'une nuit. Le roi

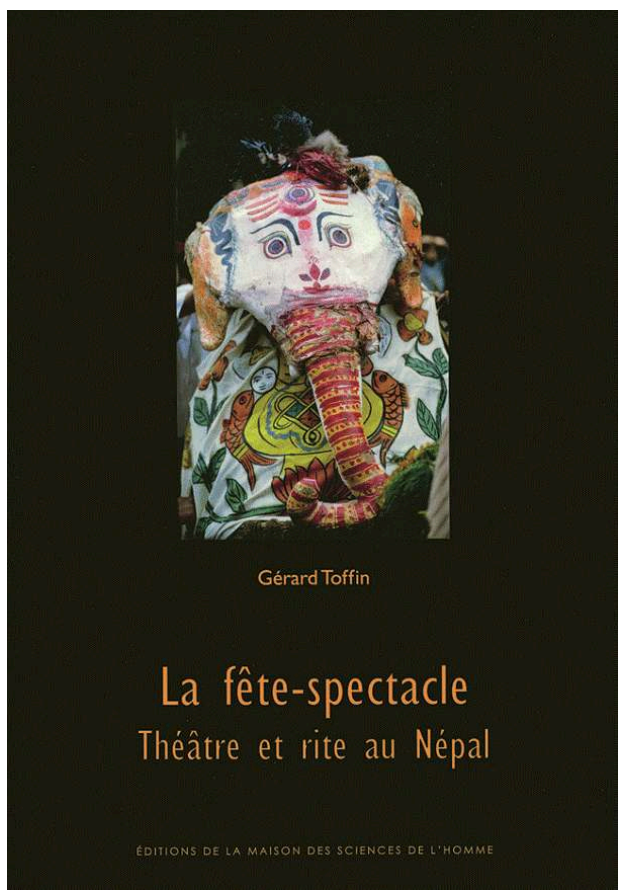
Gyanendra, qui tenta follement de faire comme son père - lequel avait interdit les formations politiques sous le régime des panchayat (1960-1990)-, ne fit que pousser les partis à s'allier aux maoïstes, à Delhi, en novembre 2005. Jusqu'alors, Prachanda n'était jamais apparu en public, si bien que certains finirent par douter de son existence. La seule photographie de lui, trouvée sur le cadavre d'un soldat rebelle, le montrait avec d'autres dirigeants maoïstes lors d'une réunion du parti, quelque part dans les collines népalaises. On imagine combien sa première interview à la BBC en 2006 fit sensation.

2 Quand Katmandou se souleva en avril-mai 2006, le roi Gyanendra, désormais incapable de gouverner seul, dut rendre leur pouvoir aux partis. Après deux ans de manœuvres politiques, des élections nationales contrôlées par des observateurs nationaux et internationaux furent organisées en avril 2008. À la stupéfaction générale, les maoïstes l'emportèrent, ne comprenant eux-mêmes leur victoire qu'au dernier moment. C'est justement parce qu'ils ne s'attendaient pas à gagner qu'ils avaient exigé que le mode de scrutin réserve une part significative à la représentation proportionnelle et qu'ils avaient essayé, mais sans succès, de faire alliance avec l'UML (les marxistes-léninistes unifiés, représentant le courant traditionnel du communisme parlementaire).

3 En définitive, une part importante de la population népalaise avait opté pour les maoïstes, mais les raisons avancées n'étaient pas toutes cohérentes : ils n'avaient pas encore été au pouvoir (le slogan maoïste «Vous avez déjà essayé plusieurs fois les autres, essayez-nous cette fois » traduisait brillamment l'humeur du jour); ils avaient souffert et méritaient récompense ; on voulait éviter qu'ils ne « reprennent le chemin de la jungle» (c'est-à-dire de la lutte armée); leur force de conviction (jos) les distinguait des politiciens usés et discrédités des autres partis.

4 Ce fut un séisme politique, non un raz de marée électoral. Les maoïstes remportèrent exactement la moitié des 240 sièges pourvus au scrutin uninominal, un succès remarquable qui leur valut un prestige considérable. Au total, ils disposèrent de 229 sièges, soit $38 \%$ de ceux-ci avec environ $30 \%$ des suffrages exprimés, ce qui faisait d'eux le premier parti du pays. Avant l'élection, leur principale ambition était de 
reléguer l'UML à la troisième place. Le parti du Congrès népalais et l'UML furent humiliés, obtenant respectivement 115 et 108 sièges dans un Parlement qui en comptait 601. Aucun parti ne pouvant compter sur 301 sièges, un gouvernement de coalition devenait inévitable.

Girija Prasad Koirala, l'indéracinable leader du parti du Congrès, dut céder en août son poste de Premier ministre aux maoïstes, qui le revendiquaient puisque détenant la majorité relative. Le parti du Congrès entra alors dans l'opposition et un gouvernement de coalition sous direction maoïste prit le relais. Le nouveau ministre des Finances, Baburam Bhattarai, était titulaire d'un doctorat de la School of Planning and Architecture obtenu à la prestigieuse université JNU de New Delhi (il est devenu Premier ministre en août 2011, bénéficiant à son tour du jeu de chaises musicales ministériel). Il s'efforça d'améliorer la collecte de l'impôt en taxant davantage les milieux d'affaires et annonça que puisque le pays, devenu laïc, n'était plus un royaume hindou, les fonds publics n'avaient plus à financer les sacrifices rituels. Ces économies s'imposaient d'autant plus que la famine régnait dans le Nord-Ouest du pays.

6 Au même moment, on célébrait Indra Jatra, l'une des fêtes les plus anciennes et les plus vénérables de la vallée de Katmandou. Indra, roi des dieux en Inde pendant la période védique, il y a plus de 2500 ans, n'a plus dans ce pays un seul temple qui lui soit consacré. Mais au Népal, dans la vallée de Katmandou, il est encore vénéré, et sa fête est l'une des plus complexes et des plus fascinantes du cycle annuel.Chacune destrois plus anciennes villes de la vallée - Katmandou, Lalitpur (Patan) et Bhaktapur - était la capitale d'un petit royaume pendant la période Malla (1200-1769). Elles ont préservé une forme ancienne de la culture de l'Asie du Sud qui remonte au premier millénaire de notre ère et ne se limite pas au culte d'Indra. La vallée apparaît ainsi comme le conservatoire de nombreux traits culturels: coexistence de l'hindouisme et du bouddhisme, omniprésence des organisations socioreligieuses appelées guthi (du sanskrit gosthi), survivance de certaines liturgies sanscrites, autrefois en usage en Asie du Sud et au-delà, et de traditions musicales et d'hymnes également très anciens. C'est ce qui a conduit le grand sanscritiste et sinologue français Sylvain Lévi à se rendre au Népal en 1898 pour écrire une histoire du pays en trois volumes qui reste un classique. Le Népal, écrit Lévi (il entendait par là la vallée de Katmandou), " c'est l'Inde qui se fait ». Par cette formule, il voulait dire que la vallée de Katmandou préserve « comme dans un laboratoire " les processus culturels et sociaux complexes qu'avait produits l'Inde médiévale (préislamique). Aujourd'hui, cette culture unique et particulièrement sophistiquée est submergée par la mondialisation, les bouleversements politiques, l'éducation et la circulation des personnes.

7 En août 2008, les nouveaux dirigeants maoïstes ne pouvaient imaginer que leur refus d'offrir trois petits buffles aux participants d'une cérémonie de la fête d'Indra Jatra provoquerait une réaction aussi spectaculaire. Ces derniers ont protesté contre la décision du gouvernement, prise sans qu'ils aient été consultés - et, selon eux, de façon inconstitutionnelle -, en refusant le lendemain de tirer les chariots des dieux. La population locale s'associa à leur protestation, et lorsque le gouvernement envoya la police et l'armée pour tirer les chariots, elle les en empêcha violemment. Pendant plusieurs jours, Katmandou fut immobilisée tandis que le gouvernement essayait de trouver un intermédiaire pour négocier avec les émeutiers.

8 Les maoïstes, assurément pris au dépourvu, mirent en cause des agents royalistes provocateurs. Le plus remarquable, c'est que les chefs ethniques du peuple néwar, 
pourtant bien connus de tous, ont disparu alors même que l'on devait célébrer leur grande fête traditionnelle. Ils n'étaient même plus joignables sur leurs téléphones portables. La plupart d'entre eux étaient sympathisants maoïstes, ou en tout cas de gauche, et beaucoup espéraient quelques faveurs de ce nouveau gouvernement. Les manifestants confectionnèrent une effigie des "chefs néwar ", qu'ils affublèrent de bracelets de femmes avant d'y mettre le feu. Ce fut, en un mot, une réaction venue véritablement du peuple néwar contre ce qui avait été perçu comme une agression - un cri de révolte contre la submersion du vieux Katmandou néwar et de ses traditions par le flot d'étrangers à la vallée, et contre un gouvernement indifférent à sa cause.

La violence de cette réaction populaire est, entre autres, au cœur du nouvel ouvrage de Gérard Toffin, un des spécialistes les plus reconnus de la culture néwar de la vallée de Katmandou. Depuis 1970, il a consacré une série d'études à des villages isolés au peuplement de caste homogène, comme Pyangaon et les Pahari marginaux de l'extrême Sud, à la ville multi-caste de Panauti, aux agriculteurs jyapu de la ville de Katmandou, et aux traditions historiques et religieuses de la royauté néwar. Il a écrit sur l'agriculture, les rituels, la parenté, les fêtes, l'architecture, la musique, l'espace, l'histoire, la royauté,... Il consacre ce nouveau livre à la fête d'Indra Jatra qu'il a observée pour la première fois à Pyangaon en 1970 et sur laquelle il est revenu à maintes reprises depuis lors.

10 Les ambitions de Gérard Toffin vont cependant bien au-delà de la seule explication des événements de 2008. Il cherche, au travers d'une étude de cas - un peu comme Émile Durkheim l'avait fait avec la cérémonie de l'Intichiuma-, à analyser de manière beaucoup plus générale la nature des fêtes religieuses, et les origines du théâtre et du drame. Il est heureux que l'on dispose sur la fête d'Indra Jatra d'éléments plus nombreux et plus fiables (car de première main) que ceux qu'avait pour sa part consultés Durkheim. Gérard Toffin cherche justement à dépasser le sociologisme durkheimien. Il a pour référence Arthur Maurice Hocart, l'auteur de The Life-Giving Myth and other Essays.Il s'oppose également à ceux qui ne voient dans les fêtes que chaos et anti-structure. Le relâchement des règles, souvent de mise en période de fête, est d'ordinaire strictement encadré. En même temps, il est vrai que les fêtes, si elles comportent de nombreuses séquences rituelles qui excluent toute déviation, jouent avec les émotions des participants d'une manière qui n'est pas entièrement prévisible. Ce n'est qu'à la fin du livre que Gérard Toffin révèle ce que les fêtes peuvent dissimuler. Dans le cas d'Indra Jatra, selon lui, c'est l'inégalité des castes et l'histoire douloureuse qui a fait de cette fête la commémoration annuelle de la défaite du peuple néwar : c'est en effet lors d'Indra Jatra qu'en 1768 le roi de Gorkha, Prithvi Narayan Shah, entra dans Katmandou pour recevoir sur le front la tika vermillon que lui apposa la déesse vivante Kumari, ce qui légitimait la prise de la ville et la chute du dernier roi néwar, Jaya Prakash Malla.

11 Gérard Toffin apporte une masse considérable d'informations sur l'énorme variété des spectacles qui composent la fête d'Indra Jatra : défilés de chariots tirés à travers la ville, exposition des dieux aux carrefours, processions rituelles, le long des vieux murs, de familles endeuillées, tableaux vivants représentant les dix avatars de Vishnu, danses masquées des dieux et des démons, interactions entre les dieux ainsi qu'entre le roi et les dieux, accompagnements musicaux, courts spectacles de marionnettes, et même représentations de scènes tirées de la mythologie ou de l'épopée, semblables aux drames que l'on jouait dans les cours médiévales. À ce propos, une représentation qui 
eut lieu jusqu'en 2002 dans le village reculé de Pyangaon le conduit à reconnaître (p. 109) que, même dans les villages les plus isolés, l'indianisation a été profonde. « Les modèles culturels de l'Inde", c'est-à-dire le drame de cour, ont été pleinement accueillis même dans des villages périphériques, peuplés de paysans de basses castes, et que, d'un point de vue structurel, Gérard Toffin avait auparavant qualifiés de «tribaux » ou « semi-tribaux ».

La thèse principale de ce livre, élégamment soutenue et largement démontrée, est que les fêtes publiques sont des spectacles aux multiples facettes qui intègrent la danse, la musique, le spectacle, pour ne rien mentionner de la nourriture et de la boisson, et au cours desquelles les participants sont autant acteurs que spectateurs. Gérard Toffin s'attache aussi à montrer que ces fêtes sont créées et modifiées - souvent, mais pas seulement, par les rois -, et que le théâtre, ou le drame dans le sens étroit du terme, tire son origine de ce contexte religieux et festif. Les premiers drames en langue néwari sont pour la plupart le fait de rois et portent précisément sur les thèmes religieux de ces fêtes.

À cet égard, Gérard Toffin aurait pu se référer aux travaux d'Horst Brinkhaus, qui a traduit deux de ces drames médiévaux néwar.Il n'a pas non plus tenté d'utiliser les œuvres en népalais et en néwari de savants locaux, qui en ont pourtant édité et traduit un grand nombre; compte tenu de sa précieuse familiarité avec la société néwar, il aurait été intéressant qu'il interroge les chercheurs locaux sur leurs travaux. Il ne dialogue pas davantage avec les autres chercheurs qui ont écrit sur les mêmes thèmes. Ainsi, l'anthropologue américain Robert Levy s'est beaucoup intéressé au «ballet civique » dans son grand œuvre, Mesocosm : Hinduism and the Organization of a Traditional City in Nepal (University of California Press, 1990). Or, si Levy est cité à quatre reprises pour des points de détail sur les coutumes de Bhaktapur, il ne l'est jamais en revanche pour son interprétation des fêtes, de l'hindouisme, du rôle des symboles qui "retiennent l'attention », ou du paysage urbain. Enfin, Sylvain Lévi, cité plus haut, est bien mentionné pour avoir écrit l'ouvrage fondateur sur les drames sanscrits, mais ses idées, à peine citées, auraient mérité d'être discutées.

Au-delà de ces réserves, qu'appelle une lecture universitaire, Gérard Toffin a écrit un livre aussi enchanteur (et très bien illustré) que l'est la fête d'Indra Jatraelle-même. Il a amplement démontré que le spectacle théâtral n'en est pas un aspect accessoire, mais au contraire constitutif et absolument indispensable. Il montre aussi que le théâtre moderne, dans le contexte du sous-continent indien (comme c'était le cas dans la Grèce antique), tire ses origines de la représentation religieuse (sans pourtant s'interroger sur la manière dont Bollywood peut avoir transposé cette tradition dans un autre registre). Gérard Toffin montre enfin comment la tradition durkheimienne (avec laquelle il ne semble pourtant pas avoir beaucoup d'affinités) peut être enrichie et approfondie. 


\section{AUTEURS}

DAVID N. GELLNER

david.gellner@anthro.ox.ac.uk 